\title{
Hyperkinetic Manifestation in a Patient with Hippocampal Atrophy
}

\author{
Almeida RD ${ }^{*}$, Regino JSP ${ }^{1}$, Costa TDA ${ }^{1}$, Ortiz AEC ${ }^{1}$, Resende LPD ${ }^{1}$, Mendonça MS ${ }^{1}$, Teixeira RM ${ }^{1}$, Lima \\ $\mathrm{BS}^{1}$, Leon $\mathrm{SA}^{2}$, and Lacerda $\mathrm{GCB}^{2}$
}

${ }^{1}$ Doctors, Specializing in Neurology, at the Gaffrée and Guinle University Hospital, at Federal University of the State of Rio de Janeiro, UNIRIO, Brazil

${ }^{2}$ Adjunct Professor of Neurology, Department of Epilepsy at the Gaffrée and Guinle University Hospital, Federal University of the State of Rio de Janeiro, UNIRIO, Brazil

${ }^{*}$ Corresponding author: Almeida RD, Doctor, Specializing in Neurology, Department of Neurology, Epilepsy Division, Federal University of Rio de Janeiro, Rua Mariz e Barros 775 Tijuca, Rio de Janeiro, 20270004, UNIRIO, Brazil, Tel: 25695925, E-mail: delfinorenata10@gmail.com

Citation: Almeida RD, Regino JSP, Costa TDA, Ortiz AEC, Resende LPD, et al. (2017) Hyperkinetic Manifestation in a Patient with Hippocampal Atrophy. J Neurol Neurol Disord 3(3): 303

Received Date: December 15, 2016 Accepted Date: August 30, 2017 Published Date: September 01, 2017

\begin{abstract}
Describes a case of a patient that presents with hippocampal atrophy leading to hyperkinetic crises of parietal-occipital origin.

Keywords: Hyperkinetic manifestation; Temporal mesial epilepsy; Hippocampal atrophy
\end{abstract}

\section{Introduction}

Temporal lobe epilepsy is the most common form of epilepsy with focal seizures in the adult population, approximately $60 \%$. It is subdivided in mesial and neocortical or lateral. Underlying pathologies include tumors, vascular malformations, cortical dysplasia, trauma and hippocampal sclerosis [1]. In literature, it is described that in mesial temporal lobe epilepsy with hippocampal sclerosis, during the crisis, occurs the presence of characteristic auras (upset malaise, fear, among others), followed by immobility, which is succeed by simple automatism, involuntary motor changes and consciousness compromise [1]. Hyperkinetic crisis is uncommon, occurring in about $3 \%$ of the cases, with changes of facial expressions, vocalization, complex motor automatisms, dystonic posturing and cephalic version. Hyperkinetic crisis indicates strong association with the symptomatogenic frontal zone, mainly orbitofrontal or medial frontal [2].

\section{Case Report}

Patient F.R.S., male, 61 years old, who at the age of 21 presented a condition of dyscognitive seizures with vocal and complex automatisms like getting undressed and walking, besides tonic seizures, with the maximum duration of 2 minutes, daily frequency, about 5 episodes a day. He could not sense the phenomenon and denied postictal alteration, like somnolence or sphincteric release. In the previous pathological history reported only mild hypertension, but did not use any antihypertensive medication, denied trauma, previous surgeries, blood transfusions or fever seizures in childhood. He had previous drug treatment with valproic acid $1500 \mathrm{mg} /$ day, which did not change the number of episodes per day, so after a few months his doctor added carbamazepine 800mg/ day but, despite he did not have side effects and had good compliance with medication, there was no clinical improvement. So, he was sent to a Drug Resistant Epilepsy Center (DREC). By the time of the appointment at the DREC, he had been taking carbamazepine $800 \mathrm{mg} /$ day and phenobarbital 200mg/day, without improvement. He brought a head MRI that showed hippocampal asymmetry (Figure 1), so it was requested a video electroencephalogram.

By the time he was hospitalized, to do the video electroencephalogram, he had normal blood pressure, was mentally stable, his carbamazepine and phenobarbital levels were inside the therapeutic range. Analysis of the video electroencephalogram: seizure originates from parietal-occipital regions bilaterally with an alpha-like rhythmic activity followed in 2 seconds by hyperkinetic manifestation waked by vocalization, a body-turning to the right, a feared face, a tonic posturing of the left and lower upper limb. The EEG begins with a rhythmic alpha-like activity, in the posterior derivations P4-O2, P3-O1 bilaterally, spreads in 2 to 3 seconds to posterior temporal derivations. The EEG is them covered by artifacts. The seizure lasts about 63 " and ends with a return to the basal interictal activity. 
As initial conduct we have chosen to reduce the use of phenobarbital and then place him on topiramate 100mg/day. It was put under increasing doses of topiramate up to $200 \mathrm{mg} /$ day and phenobarbital was waned off at a $25 \mathrm{mg} /$ day rate every 10 days. He progresses with sudden drops to the frequency of twice a week, besides the dyscognitive seizures. Patient's compliance was achieved and he turned out to be seizure-free in about 4 weeks. He is still been followed, in the Drug Resistant Epilepsy Center, monthly, and it has 4 months that he is seizure-free.

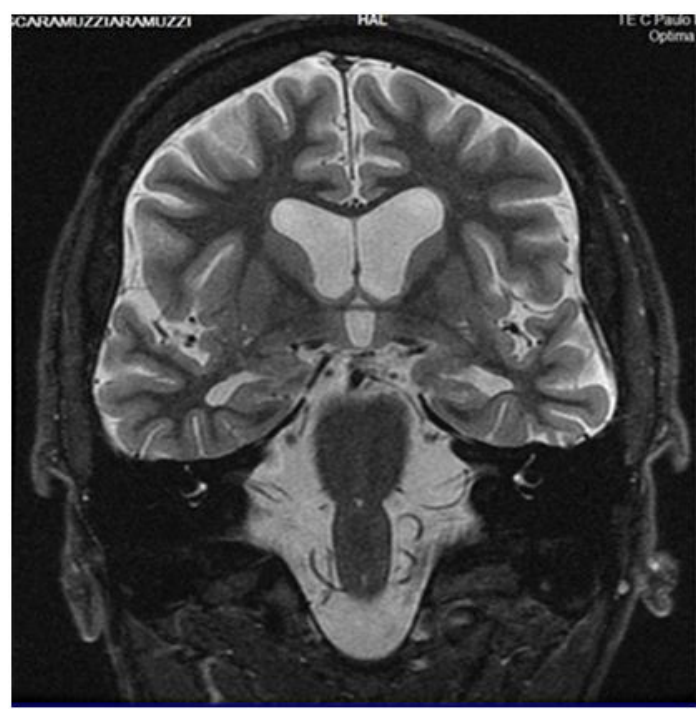

Figure 1: Magnetic resonance imaging, which shows hippocampal asymmetry that may correspond to left mesial temporal sclerosis

\section{Discussion}

Currently, hypermotor seizure (HMS) is defined as bizarre, complex and high-amplitude movements, involving proximal segment and resulting in a violent and inappropriate movement. It occurs in 15 to $27 \%$ of frontal lobe epilepsies, particularly in the mesial area, or orbitofrontal, but it was also described in seizures originating in the dorsolateral region, in mesial region and neocortical of the temporal lobe, upper and anterior portions of the insular cortex and the parietal-temporal cortex (Figure 2A and 2B) [3,4].
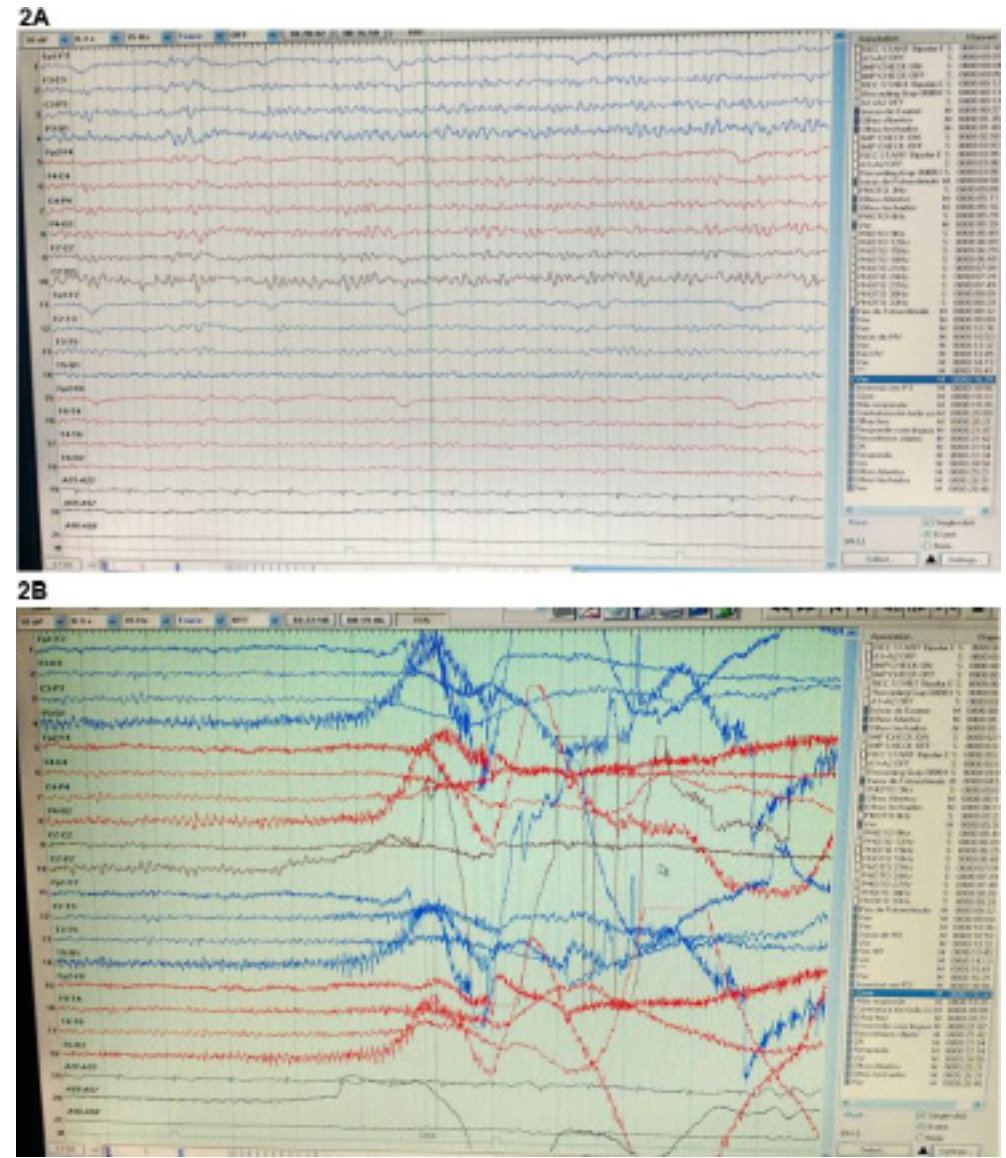

Figure 2A and B: High waves with phase inversion in P3 or equipotential in T3-P3 and rhythmic activity at $8 \mathrm{~Hz}$ in the parietal-temporal-occipital regions, on both sides, radiating to other leads 


\section{https://www.youtube.com/watch?v=Vkk0SUoK58c}

It is subdivided in 2 types: type HMS1, in which axial movements occur, that is movement around a vertical axis, while the semiology of type HMS2 occurs on a horizontal axis. HMS1 is associated to a epileptogenic zone in the ventromedial frontal cortex and HMS2 has been associated with a dorsal epileptogenic zone [4,5]. The clinical differentiation consists of: type HMS1 has marked agitation and hypermotor behaviors with kicks, punches, swinging body and other motor signs, such as versive head movements, non-motor related signals, like facial expression of fear or anger, and dysautonomic symptoms. Subtype HMS2: a less intense agitation occurs with hypermotor behavior (trunk and pelvis rotation and other motor signals such as diatonic posture and versive movements of the head) [5].

A mixed type of HMS1 and HMS2 was described in insular seizures, characterized mainly by repetitive pelvic, bimanual and bipedal movements, including pelvic thrusts and kicking.

HMS typically occurs at, or within 10 seconds after, the onset of the seizure. The average delay of HMS after the onset of the seizures helps distinguish the extrafrontal origin of HMS. The average delay between 4 and 30 seconds occurs in temporal epilepsy, 5 to 40 seconds in insular seizures and 10 to 35 seconds in posterior HMS [6].

Surgery in the posterior cortex is rarely performed, comparing to resections of the temporal and frontal lobes. The main reason is the difficulty in identifying the epileptogenic zone in the pre-operative assessment [6,7].

The International League against Epilepsy defined drug resistant epilepsy, as "a failure of equate trials of two tolerated, appropriately chosen and used anticonvulsant drug schedules (whether as monotherapies or in combination) to achieve sustained seizure freedom" [8].

First-generation or "old" AEDs such as carbamazepine, phenobarbital and phenytoin are enzyme inducers. Same examples of second-generation or "newer" AEDs are lamotrigine, gabapentin, topiramate. Carbamazepine, phenytoin, phenobarbital and primidone are broad- spectrum inducers because they stimulate the activity of many cytochrome P45 (CYP) enzymes as well as uridin-glucuronyl transferases (UGT) and epoxide hydrolase.

Oxcarbazepine, eslicarbazepine acetate, felbamate, rufinamide, topiramate (at doses $>200 \mathrm{mg} /$ day) and perampanel ate doses $>8 \mathrm{mg}$ /day) have weaker enzyme-inducing properties and may stimulate a more restricted range of CYP and/or UGT isoenzymes [9].

\section{Conclusion}

A report was made of a patient with hippocampal atrophy that presented type 2 hyperkinetic seizures (usually a frontal manifestation) with epileptogenic discharge in the parietal-occipital region.

\section{References}

1. Salzmann A, Malafosse A (2012) Genetics of Temporal Lobe Epilepsy: A Review. Epilepsy Res Treat 2012: 19.

2. Staack AM, Bilic S, Wendling AS, Scholly J, Kraus U, et al. (2011) Hyperkinetic seizures in patients with temporal lobe epilepsy: clinical features and outcome after temporal lobe resection. Epilepsia 52: 1439-46.

3. Montavont A, Kahane P, Catenoix H, Ostrowsky-Coste K, Isnard J, et al. (2013) Hypermotor seizures in lateral and mesial parietal epilepsy. Epilepsy Behav 28: 408-12.

4. Alqadi K, Sankaraneni R, Thome U, Kotagal P (2016) Semiology of hypermotor (hyperkinetic) seizures. Epilepsy Behav 54: 137-41.

5. Andrade-Machado R (2016) Understanding Hyper Motor Seizures. J Epilepsy 2: 108.

6. Yu T, Wang Y, Zhang G, Cai L, Du W, et al. (2009) Posterior cortex epilepsy: diagnostic considerations and surgical outcome. Seizure 18: 288-92.

7. Tao Y, Guojun Z, Yuping W, Lixin C, Wei D, et al. (2010) Surgical treatment of patients with drug-resistant hypermotor seizures. Epilepsia 51: 2124-30.

8. Nair DR (2016) Management of Drug-Resistant Epilepsy. Continuum (Minneap Minn) 22: 157-72.

9. Jacob S, Nair AB (2016) An Updated Overview on Therapeutic Drug Monitoring of Recent Antiepileptic Drugs. Drugs R D 16: $303-16$. 


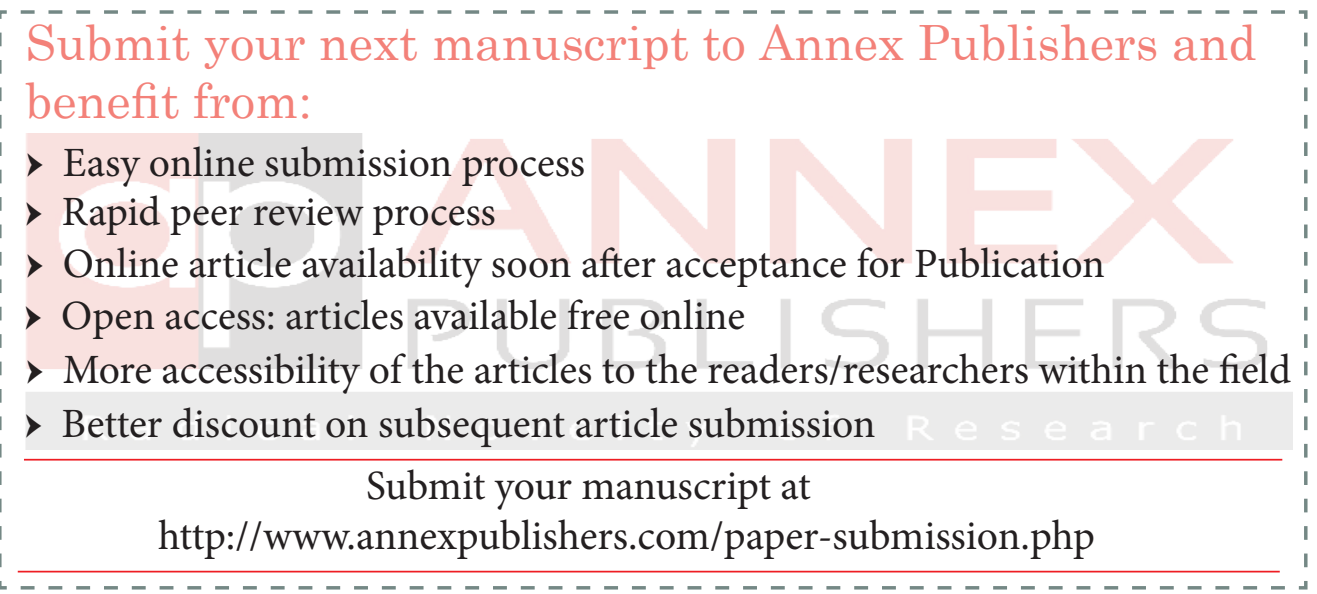

